\title{
COLLINEATIONS AND GENERALIZED INCIDENCE MATRICES
}

\author{
BY \\ D. R. HUGHES(1)
}

1. Introduction. In an earlier paper [12] the author has found certain numerical conditions which must be satisfied if a $(v, k, \lambda)$ configuration is to possess a regular collineation group. These conditions were derived from relations similar to the incidence matrix equations of $[3 ; 4]$. Here these conditions are extended in that we show the existence of certain matrix equations which must be satisfied if a $(v, k, \lambda)$ configuration is to possess an arbitrary collineation group. Using these equations, it is shown that the number of transitive classes of points is always equal to the number of transitive classes of lines $\left({ }^{2}\right)$, and that the matrix equations always lead to a rational congruence.

Specializing the collineation groups considered to the class of standard collineation groups, we apply the Hasse-Minkowski theory to the rational congruence mentioned above and derive numerical conditions on the parameters $v, k, \lambda$, the order $m$ of the collineation group, and the number $N$ of fixed points. A standard collineation group is one whose nonidentity elements all fix the same set of points and lines; any collineation group of prime order is standard, so a $(v, k, \lambda)$ configuration with nontrivial collineations possesses nontrivial standard collineation groups. The author wishes to take this opportunity to thank H. J. Ryser for his helpful comments in the preparation of this part of the paper, in particular with the application of the HasseMinkowski theory.

Several interesting but unsolved problems in the theory of groups arise from these investigations. An intimate connection is displayed between the theories of collineations of $(v, k, \lambda)$ configurations, groups possessing subsets similar to partial difference sets, and topics in the theory of matrices with integer elements.

2. Collineation groups. Let $v, k, \lambda$, where $v>k>\lambda>0$, be integers satisfying $\lambda(v-1)=k(k-1)$. Let $\pi$ be a collection of $v$ points and $v$ lines, together with an incidence relation (i.e., point on line, line contains point, etc.) such that each point (line) is on $k$ lines (contains $k$ points) and such that each pair of distinct points (lines) are on exactly $\lambda$ common lines (contain exactly $\lambda$

Presented to the Society, August 24, 1956; received by the editors April 25, 1956 and, in revised form, July 27, 1956.

(1) This research was carried out while the author was a National Science Foundation Fellow.

(2) In a paper to appear in the Proceedings of the American Mathematical Society, E. T. Parker shows that the number of transitive classes of points equals the number of transitive classes of lines, using entirely different methods of proof, and indeed shows even more about collineations of $(v, k, \lambda)$ collineations. 
common points). ('These axioms are redundant, a fact which is of no interest here; see $[4 ; 7 ; 15]$ for further discussion.) Then $\pi$ is a $(v, k, \lambda)$ configuration (or a symmetric balanced incomplete block design, or a $\lambda$-plane); if $\lambda=1$ then $\pi$ is a finite projective plane with $v=n^{2}+n+1, k=n+1$. In general, we define the order $n$ of $\pi$ as $n=k-\lambda$. A collineation of $\pi$ is a one-to-one mapping of points onto points, lines onto lines, which preserves incidence.

Let $(B)$ be a collineation group of $\pi$, and let $m$ be the order of $(S$. The points of $\pi$ can be broken up into $w_{1}$ transitive classes $P_{i}, i=1,2, \cdots, w_{1}$, where $P$ and $Q$ are in the same transitive class if and only if $Q=P x$ for some $x$ in (5). Similarly, the lines of $\pi$ can be broken up into $w_{2}$ transitive classes $d_{i}, i$ $=1,2, \cdots, w_{2}$. Throughout this paper we shall use "class" as a synonym for "transitive class" (with respect to $(\$)$ ), if no ambiguity results. In each class $P_{i}$ choose a "base point" $P_{\imath}$ and in each class $\mathcal{J}_{i}$ a "base line" $J_{i}$. Let $\mathfrak{P}_{i}$ be the subgroup of (B) which fixes $P_{i}$ and let $\Im_{i}$ be the subgroup of (S) which fixes $J_{i}$; let $\mathfrak{B}_{i}$ have order $r_{i}$ and let $\Im_{i}$ have order $s_{i}$. Then each point in $\mathcal{P}_{i}$ is fixed by a subgroup of order $r_{i}$ and each line in $\mathscr{J}_{i}$ is fixed by a subgroup of order $s_{i}$. Let $D_{i j}$ be the subset of $B$ consisting of all $x$ such that $P_{i} x$ is on $J_{j}$, and let $a_{i j}$ be the number of elements in $D_{i j}$. Note that $\mathfrak{B}_{i} D_{i j} \Im_{j}=D_{i j}$, and so both $r_{i}$ and $s_{j}$ divide $a_{i j}$.

THEOREM 2.1. (i) $\sum_{j} a_{i j} / s_{j}=\sum_{j} a_{j i} / r_{j}=k$, for each $i=1,2, \cdots, w_{1}$, or $i=1,2, \cdots, w_{2}$, as appropriate.

(ii) $\sum_{j} a_{i j}^{2} / s_{j}=r_{i} n+\lambda m$, for each $i=1,2, \cdots, w_{1}$, and $\sum_{j} a_{j_{i}}^{2} / r_{j}=s_{i} n$ $+\lambda m$, for each $i=1,2, \cdots, w_{2}$.

(iii) For each $i, j=1,2, \cdots, w_{1}, i \neq j, \quad \sum_{t} a_{i t} a_{j t} / s_{t}=\lambda m$, and for each $i, j=1,2, \cdots, w_{2}, i \neq j, \sum_{t} a_{t i} a_{t j} / r_{t}=\lambda m$.

Proof. (i) Each line of $\partial_{j}$ which contains $P_{i}$ is counted exactly $s_{j}$ times by $a_{i j}$, hence $\sum_{j} a_{i j} / s_{j}=k$; the other half of (i) is similar, counting points on the line $J_{i}$.

(ii) Let $i$ be fixed, where $i$ is one of $1,2, \cdots, w_{1}$. If $x \in\left(G, x \notin \Re_{i}\right.$, then $P_{i} \neq P_{i} x$, so there are exactly $\lambda$ choices of $j$ (not all necessarily distinct) such that $P_{i}, P_{i} x$ are both on $J_{j} y$, for some $y$. But for each $j$ and $y$, there are in fact $s_{j}$ choices of $y$. Hence $y^{-1}, x y^{-1} \in D_{i j}$, or $x=d_{1} d_{2}^{-1}$, where $d_{1}, d_{2} \in D_{i j}$, holds for $\lambda$ choices of $j$ and $s_{j}$ choices of the pair $d_{1}, d_{2} \in D_{i j}$ for each such $j$; note that $d_{1} \in \mathfrak{P}_{i} d_{2}$. Conversely, if $d_{1} \in \mathfrak{B}_{i} d_{2}$, where $d_{1}, d_{2} \in D_{i j}$, then $d_{1}, d_{2}$ determine $x=d_{1} d_{2}^{-1} \in \mathfrak{P}_{i}$. Hence $\sum_{j} a_{i j}\left(a_{i j}-r_{i}\right) / s_{j}$ counts the number of elements of $\mathcal{B}$, each $\lambda$ times, excepting the elements of $\mathfrak{B}_{i}$. So $\sum_{j} a_{i j}\left(a_{i j}-r_{i}\right) / s_{j}$ $=\lambda\left(m-r_{i}\right)$. Hence, using (i), $\sum_{j} a_{i j}^{2} / s_{j}=r_{i} k+\lambda m-r_{i} \lambda=r_{i} n+\lambda m$. The other half of (ii) is similar, using the pair of lines $J_{i}, J_{i} x, x \in \Im_{i}$, to find representations $x=d_{1}^{-1} d_{2}$, where $d_{1}, d_{2} \in D_{j i}$.

(iii) Let $i, j$ be chosen from among $1,2, \cdots, w_{1}$, where $i \neq j$. For each $x \in B S, P_{i}$ and $P_{j} x$ determine $\lambda$ lines $J_{t} y$, whence as in (ii), $x=d_{1} d_{2}^{-1}$, where $d_{1} \in D_{j t}, d_{2} \in D_{i t}$, for $\lambda$ choices of $t$ and $s_{t}$ choices of $d_{1}, d_{2}$ for each $t$. Thus the 
first half of (iii) follows immediately, and the other half is analogous.

Now let $C_{1}$ be the square diagonal matrix of order $w_{2}$ with $s_{i}^{-1}$ in its $i$ th diagonal position; let $C_{2}$ be the square diagonal matrix of order $w_{1}$ with $r_{i}^{-1}$ in its $i$ th diagonal position. Let $B_{1}$ be the square matrix of order $w_{1}$ with $\lambda m$ in all positions off of the main diagonal and $r_{i} n+\lambda m$ in the $i$ th diagonal position; let $B_{2}$ be the square matrix of order $w_{2}$ with $\lambda m$ in all positions off of the main diagonal and $s_{i} n+\lambda m$ in the $i$ th diagonal position. Let $A$ be the matrix $\left(a_{i j}\right)$. Throughout the paper we shall let $E^{T}$ denote the transpose of the matrix $E$, and let det $(E)$ denote the determinant of $E$, if $E$ is square. Then Theorem 2.1 can be rephrased in matrix form as follows:

Theorem 2.2. $A C_{1} A^{T}=B_{1}, A^{T} C_{2} A=B_{2}$; each row sum of $A C_{1}$ and each column sum of $C_{2} A$ is $k$.

Now suppose $w_{1}>w_{2}$. Then the matrix $A C_{1}$ can be made into a square matrix $A_{1}$ of order $w_{1}$ by adjoining $w_{1}-w_{2}$ columns of zeros on its right, and the matrix $A^{T}$ becomes a square matrix $A_{2}$ of order $w_{1}$ by adjoining $w_{1}-w_{2}$ rows of zeros beneath it. But $A_{1} A_{2}=B_{1}$, whence $B_{1}$ must be singular, as both $A_{1}$ and $A_{2}$ are. Similarly, if $w_{2}>w_{1}$, then there are singular square matrices $A_{3}, A_{4}$ such that $A_{3} A_{4}=B_{2}$, whence $B_{2}$ is singular.

Lemma 2.1. Let $B$ be a square matrix of order $w$ with $b_{i}+d$ in the ith position on the main diagonal, $b_{i} \neq 0$, and $d$ elsewhere. Then $\left({ }^{3}\right)$

$$
\operatorname{det}(B)=\prod_{i=1}^{i-w} b_{i}+d\left[\sum_{i=1}^{i=w}\left(\prod_{i=1}^{i=w} b_{j}\right) / b_{i}\right] .
$$

Proof. Subtract the last column of $B$ from every other column; the resulting matrix $B^{\prime}$ has the same determinant as $B$. In the lower right corner of $B^{\prime}$ is the element $b_{w}+d$, the rest of the last column consists entirely of $d$, the rest of the last row consists entirely of $-b_{w}$, and the rest of $B^{\prime}$ is a diagonal matrix of order $w-1$ with $b_{1}, b_{2}, \cdots, b_{w-1}$ down its main diagonal.

Now we prove the lemma by induction. It is clearly true if $w=1$, so we assume it true for $w-1$. Let $B$ have order $w$ and consider $B^{\prime}$ as defined above. It is easy to see that $\operatorname{det}(B)=\operatorname{det}\left(B^{\prime}\right)=b_{1} \operatorname{det}\left(D_{1}\right)+(-1)^{w-1} d \operatorname{det}\left(D_{2}\right)$, where

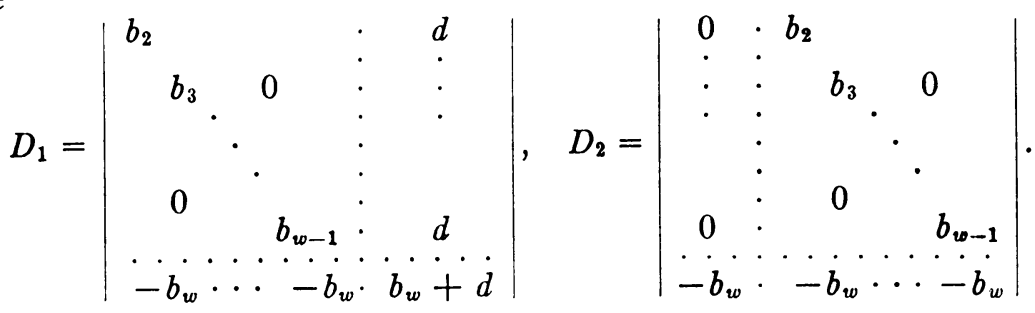

(3) If $b_{1}$, say, is zero, then $\operatorname{det}(B)=d \prod_{-2}^{\infty} b_{i}$, while $B$ is singular if as many as two of the $b_{i}$ are zero. 
But by the induction hypothesis,

$$
\operatorname{det}\left(D_{1}\right)=\prod_{i=2}^{i=w} b_{i}+d\left[\sum_{i=2}^{i=w}\left(\prod_{i=2}^{i=w} b_{i}\right) / b_{i}\right]
$$

Also, it is easy to compute $\operatorname{det}\left(D_{2}\right)$ :

$$
\operatorname{det}\left(D_{2}\right)=(-1)^{w}\left(-b_{u}\right) \prod_{i=2}^{w-1} b_{i}
$$

Combining these, it is immediate that the formula of the lemma is correct.

Since the matrices $B_{1}$ and $B_{2}$ consist of positive entries, they are both nonsingular. So:

THEOREM 2.3. If (i) is a collineation group of the $(v, k, \lambda)$ configuration $\pi$, then the number of transitive classes of points of $\pi$ equals the number of transitive classes of lines of $\pi$ (with respect to (\$). The matrix $A$ of Theorem 2.2 is square and nonsingular.

From Lemma 2.1 we can derive some further results. Let $w=w_{1}=w_{2}$, and let $r=\prod r_{i}, s=\prod s_{i}$, where the products are from 1 to $w$. Then where all sums are from 1 to $w$, det $\left(B_{1}\right)=s n^{w-1}\left(n+\lambda m \sum s_{i}^{-1}\right)$. However, $m / s_{i}$ is the number of lines in the class $\mathcal{I}_{i}$, so $m \sum s_{i}^{-1}=v$. Since $\lambda(v-1)=k(k-1)$ implies $n+\lambda v=k^{2}$, this yields:

$$
\operatorname{det}\left(B_{1}\right)=s n^{w-1}(n+\lambda v)=s n^{w-1} k^{2} .
$$

Now, $\operatorname{det}\left(C_{1}\right)=1 / r$, and so $[\operatorname{det}(A)]^{2}=r s n^{w-1} k^{2}$. The other matrix equation leads to the same result. Thus we have proved:

THEOREM 2.4. If $r, s, w$ are defined as above, then $r s n^{w-1}$ is a square.

Now let $S$ be the square matrix of order $w$, all of whose entries are +1 . Then Theorem 2.2 can be stated as $A C_{1} A^{T}=n C_{2}^{-1}+\lambda m S, A^{T} C_{2} A=n C_{1}^{-1}$ $+\lambda m S$, and $A C_{1} S=S C_{2} A=k S$.

THEOREM 2.5. If $D_{1}$ is the diagonal matrix of order $w$ with $x_{i}$ in the ith diagonal position, where each $x_{i}$ is a nonzero real number, if $X$ and $D_{2}$ are nonsingular matrices of order $w$, and if $n, k, y$ are reals satisfying $n \neq 0, k \neq 0$, $y \sum_{i} x_{i}=k^{2}-n$, then

(i) $\quad X D_{1} X^{T}=n D_{2}^{-1}+y S$

$$
X D_{1} S=k S,
$$

implies

(iii) $X^{T} D_{2} X=n D_{1}^{-1}+y S$,

$$
S D_{2} X=k S .
$$

Proof. From (i) we have $D_{1}=n\left(X^{T} D_{2} X\right)^{-1}+y X^{-1} S\left(X^{-1}\right)^{T}$; but from (ii), $X^{-1} S=k^{-1} D_{1} S \quad$ and $\quad S\left(X^{-1}\right)^{T}=\left(X^{-1} S\right)^{T}=k^{-1} S D_{1}, \quad$ so $\quad\left(X^{T} D_{2} X\right)^{-1}$ $=n^{-1}\left(D_{1}-y k^{-2} D_{1} S D_{1}\right)$. Let $P=\left(X^{T} D_{2} X\right)^{-1}\left(n D_{1}^{-1}+y S\right)$ and let $I$ be the iden- 
tity matrix of order $w$. Then:

$$
\begin{aligned}
P & =I+n^{-1} y D_{1} S-y k^{-2} D_{1} S-y^{2} k^{-2} n^{-1}\left(D_{1} S\right)^{2} \\
& =I+y n^{-1} k^{-2}\left[\left(k^{2}-n\right) D_{1} S-y\left(D_{1} S\right)^{2}\right] .
\end{aligned}
$$

But $D_{1} S$ is a square matrix of order $w$ whose $i$ th row consists completely of $x_{i}$, and if we let $b=\sum_{i} x_{i}$, then $\left(D_{1} S\right)^{2}=b D_{1} S$. Since $y b=k^{2}-n$ :

$$
P=I+y n^{-1} k^{-2}\left(k^{2}-n-y b\right) D_{1} S=I,
$$

and we have proved (iii).

From (iii), $D_{2} X=n\left(X^{-1}\right)^{\boldsymbol{T}} D_{1}^{-1}+y\left(X^{-1}\right)^{T} S$, so $S D_{2} X=n S\left(X^{-1}\right)^{T} D_{1}^{-1}$ $+y S\left(X^{-1}\right)^{T} S$. As above, $S\left(X^{-1}\right)^{T}=k^{-1} S D_{1}$, so $S D_{2} X=n k^{-1} S+y k^{-1} S D_{1} S$. The product $S\left(D_{1} S\right)$ "sums the columns" of $D_{1} S$, and so $S D_{1} S=b S$. Hence $S D_{2} X$ $=k^{-1}(n+y b) S=k^{-1}\left(k^{2}\right) S=k S$, which is (iv).

Clearly the necessary "converse" of Theorem 2.5 can be proved, whence if $C_{i}, B_{i}, i=1,2$, are the matrices of Theorem 2.2, we have the following:

Corollary. The square matrix $X$ satisfies (i) if and only if it satisfies (ii):

(i) $X C_{1} X^{T}=B_{1}, \quad X C_{1} S=k S$;

(ii) $X^{T} C_{2} X=B_{2}, \quad S C_{2} X=k S$.

Hence we need concern ourselves with only half of Theorem 2.2 , if we are merely interested in the existence or nonexistence of the matrix $A=\left(a_{i j}\right)$.

3. Standard collineations. Suppose $\&$ is a collineation group of the $(v, k, \lambda)$ configuration $\pi$, and as above let $m$ be the order of $\$$. If every nonidentity element of $\&$ fixes the same set of points and lines of $\pi$, then we say that $(B)$ is a standard collineation group; note that this is equivalent to demanding that every $r_{i}$ and $s_{i}$ be either 1 or $m$. Let $N$ be the number of points fixed by (B), and let $N_{1}$ be the number of lines fixed. The number of transitive classes of points is $(v-N) / m+N$, while the number of transitive classes of lines is $\left(v-N_{1}\right) / m+N_{1}$; since these two expressions must be equal, we have $N=N_{1}$.

The matrix $C_{1}$ has $t=(v-N) / m$ ones on its main diagonal and we can assume that these are in the first $t$ positions; the remaining $N$ elements on the main diagonal are all $1 / m$. The matrix $B_{1}$ has $t$ elements $n+\lambda m$ on its main diagonal, which we can also assume to be in the first $t$ position; then the remaining $N$ diagonal elements are $m n+\lambda m$. Let $C^{\prime}=C_{1}, B=B_{1}$.

The classical theory of rational congruence asserts that if $A, B, C$ are nonsingular matrices $\left(^{4}\right)$ of the same order, consisting entirely of rational entries, and if $A C A^{T}=B$, then the "Hasse invariants" of $B$ and $C$ are equal (and of course, $\left.[\operatorname{det}(A)]^{2}[\operatorname{det}(C)]=\operatorname{det}(B)\right)$. Although this theory applies to the more general matrix relations of Theorem 2.2 , we shall confine our attention to the standard collineation group situation. Since there exists a rational nonsingular matrix $D$ such that $D C^{\prime} D^{T}=C$, where $C$ is the diagonal matrix with

$\left.{ }^{4}\right) B$ and $C$ must be symmetric, and this assumption is tacitly made at all relevant points in the discussion which follows. 
one in the first $t$ diagonal positions and $m$ in the last $N$ positions, we can assume that $C^{\prime}$ has been replaced by the integral matrix $C$. Furthermore, $r=s$, so Theorem 2.4 asserts that $n^{t+N-1}$ is a square; i.e., either $t+N-1$ is even or $n$ is a square.

We now proceed to a description of the relevant points of the HasseMinkowski theory of rational congruence; the reader is referred to $[13$, Chap. II] for proofs and a more thorough discussion. Let $C \cong B$ mean that $C$ and $B$ are rationally congruent matrices: that is, $C$ and $B$ are nonsingular matrices of the same order, with rational entries, for which $A C A^{\boldsymbol{T}}=B$ for some matrix $A$ with rational entries. If $x$ and $y$ are nonzero rational numbers such that $x / y$ is a rational square, then we write $x \cong y$.

First we discuss the Hilbert norm-residue symbol (Hilbert symbol, for short) $(x, y)_{p} ;(x, y)_{p}$ is defined for all nonzero rational numbers $x$ and $y$, and for all primes $p$. Where no ambiguity is possible, we shall omit the subscript $p$ on the Hilbert symbol. Some of the properties of the Hilbert symbol are given below, where it is understood that $x, y, z$ are nonzero rational numbers:

(1) $(x, y)_{p}=(y, x)_{p}$

(2) $(x, y)_{p}(x, z)_{p}=(x, y z)_{p}$;

(3) $(x, y)_{p}=\left(x, y z^{2}\right)_{p}$;

(4) if $n, n_{1}, m$ are nonzero integers and $n \equiv n_{1} \neq \equiv(\bmod p)$, then $(n, m)_{p}$ $=\left(n_{1}, m\right)_{p}$

(5) if $n$ is an integer, $n \neq 0,1$, then $(n, n)_{p}=(n, n-1)_{p}=(n,-1)_{p}$;

(6) if $n$ and $m$ are nonzero integers, if $p$ is an odd prime, and if $n=p^{a} n_{1}$, $m=p^{b} m_{1}$, where $n_{1}$ and $m_{1}$ are prime to $p$, then $(n, m)_{p}=(-1 \mid p)^{a b}\left(n_{1} \mid p\right)^{b}$ $\left(m_{1} \mid p\right)^{a}$, where $(x \mid p)$ is the Legendre symbol.

Now let $D$ be a square nonsingular matrix of order $u$, consisting of rational entries, and let $D_{i}$ be the determinant of the $i$ th ordered submatrix in the upper left corner of $D$. For our purposes we can assume that all of the $D_{i}$ are nonzero. Then for each prime $p$ the Hasse invariant $c_{p}(D)$ is defined by:

$$
c_{p}(D)=\left(-1, D_{u}\right)_{p} \prod_{i=1}^{u-1}\left(D_{i},-D_{i+1}\right)_{p}
$$

Then $B \cong C$ if and only if $c_{p}(B)=c_{p}(C)$ for all primes $p$, and $\operatorname{det}(B)$ $\cong \operatorname{det}(C)$.

Let $a, b$ be rational numbers, $u$ a positive integer, and define an $(a, b, u)$ matrix to be a square matrix of order $u$ with $a+b$ on its main diagonal and $b$ elsewhere.

Lemma 3.1. If $a, b$ are integers and $B$ is an $(a, b, u)$-matrix, then $\operatorname{det}(B)$ $=a^{u-1}(a+b u)$. If $B$ is nonsingular and if $p$ is an odd prime, then $c_{p}(B)$ $=(a,-1)_{p}^{u(u-1) / 2}(a, u)_{p}\left(-u a^{u-1}, a+b u\right)_{p}$. 
Proof. The first part of the lemma follows from Lemma 2.1. To prove the second part, subtract the last column of $B$ from every other column, then subtract the last row from every other row. This yields a matrix $D \cong B$, where $D$ has $a+b$ in the lower right corner, $-a$ in the rest of the last row and last column, and the rest of $D$ has $a$ off of the main diagonal and $2 a$ on the main diagonal. Letting $D_{i}$ be the determinant of the $i$ th ordered submatrix of $D$ in the upper left corner, we have:

$$
D_{i}=a^{i}(i+1) \text { for } i=1,2, \cdots, u-1, \text { and } D_{u}=a^{u-1}(a+b u) .
$$

Then:

$$
\begin{aligned}
\prod_{i=1}^{u-2}\left(D_{i},\right. & \left.-D_{i+1}\right)=\prod_{i=1}^{u-2}\left(a^{i}(i+1),-a^{i+1}(i+2)\right) \\
& =\prod_{i=1}^{u-2}\left(a^{i},-a^{i+1}\right) \prod_{i=1}^{u-2}\left(a^{i+1}, i+1\right) \prod_{i=1}^{u-2}\left(a^{i+2}, i+2\right) \prod_{i=1}^{u-2}(i+1,-(i+2)) \\
& =(a,-1)^{e}\left(a^{2}, 2\right)\left(a^{u}, u\right) \prod_{i=1}^{u-2}(i+1,-1) \prod_{i=1}^{u-2}(-1, i+2) \\
& =(a,-1)^{\epsilon}\left(-a^{u}, u\right),
\end{aligned}
$$

where $\epsilon=(u-1)(u-2) / 2$, and where we are assuming that $p$ is odd; thus $(2,-1)_{p}=+1$, for instance. So:

$$
\begin{aligned}
c_{p}(D) & =\left(-1, a^{u-1}(a+b u)\right)\left(a^{u-1} u,-a^{u-1}(a+b u)\right)(a,-1)^{\bullet}\left(-a^{u}, u\right) \\
& =(a,-1)^{u(u-1) / 2}(a, u)\left(-u a^{u-1}, a+b u\right),
\end{aligned}
$$

and the lemma is proved.

Now we return to the matrices $B$ and $C$ of the standard collineation group situation. If $N=0$, then the group (s) is in fact regular and this case has been treated in [12]; if $N=v$, then $m=1$ and the relevant matrix equations are the incidence matrix equations of [4]. So we assume that $N \neq 0, v$ in what follows. It is easy to see that $c_{p}(C)=(m,-1)^{N(N+1) / 2}$, so we have $c_{v}(B)$ to compute.

Sum the last $N$ rows of $B$, multiply by $\lambda /(n+\lambda N)$, and subtract from every other row; then sum the last $N$ columns, multiply by $\lambda /(n+\lambda N)$, and subtract from every other column. The result of these operations is a matrix $B^{\prime} \cong B$, where $B^{\prime}=B_{1}^{*} \oplus B_{2}$, and where $B_{1}^{*}$ is an $(n, \lambda m n /(n+\lambda N), t)$-matrix, $B_{2}$ is an $(m n, \lambda m, N)$-matrix ( $\oplus$ " denotes matrix direct sum). Furthermore, $B_{1}^{*} \cong B_{1}$, where $B_{1}$ is an $\left(n(n+\lambda N)^{2}, \lambda m n(n+\lambda N), t\right)$-matrix. We need the following lemma, whose proof will be found in [13]:

Lemma 3.2. If $D=D_{1} \oplus D_{2}$, where each $D_{i}$ is rational and nonsingular, and if $d_{i}=\operatorname{det}\left(D_{i}\right), i=1,2$, then $c_{p}(D)=c_{p}\left(D_{1}\right) c_{p}\left(D_{2}\right)\left(d_{1}, d_{2}\right)_{p}$.

Since $n(n+\lambda N)^{2}+\lambda m n(n+\lambda N) t=n(n+\lambda N)(n+\lambda v)=n(n+\lambda N) k^{2}$, we have: 


$$
\begin{aligned}
c_{p}\left(B_{1}\right) & =(n,-1)^{t(t-1) / 2}(n, t)\left(-t n^{t-1}, n(n+\lambda N)\right) \\
& =(n,-1)^{t(t+1) / 2}\left(n+\lambda N,-t n^{t-1}\right),
\end{aligned}
$$

and also, $\operatorname{det}\left(B_{1}\right) \cong n^{t}(n+\lambda N)$.

Similarly, $c_{p}\left(B_{2}\right)=(m,-1)^{N(N+1) / 2}(n,-1)^{N(N-1) / 2}(n, N)(m, n)^{N-1}\left(n+\lambda N,-N(m n)^{N-1}\right)$, and $\operatorname{det}\left(B_{2}\right) \cong n^{N-1} m^{N}(n+\lambda N)$.

Now we shall understand that $(+1)^{1 / 2}=+1$; then $\left(x^{2}, y\right)^{1 / 2}=+1$, for instance. Furthermore, $(n, x)^{t+N-1}=+1$ for all $x$, since either $n$ is a square or $t+N-1$ is even. Now from the above expressions for $c_{p}\left(B_{i}\right)$ and $\operatorname{det}\left(B_{i}\right)$, we have, using Lemma 3.2 and simplifying:

$$
c_{p}(B)=(m,-1)^{N(N+1) / 2}(n,-1)^{\epsilon}(n, m)^{N-1}(n, N)(n+\lambda N,-n N(v-N)),
$$

where $\epsilon=t(t+1) / 2+(N-1)(N-2) / 2$. But either $n$ is a square, or $\epsilon \equiv(t+N-1) / 2(\bmod 2)$, so we can replace $\epsilon$ by $(t+N-1) / 2$.

Lemma 3.3. For odd primes $p,(n, N)_{p}(n+\lambda N,-n N(v-N))_{p}=(n, \lambda)_{p}$.

Proof. The proof is extremely tedious and long, but is rather straightforward, relying highly on property (6) of the Hilbert symbol. We will give the complete proof for the case $n \neq 0(\bmod p)$, since this amply demonstrates the technique, and only sketch the case $n \equiv 0(\bmod p)$. For the rest of the proof, all congruences are modulo $p$. Let $f=(n, \lambda N)(n+\lambda N,-n N(v-N))$; we must show that $f=+1$.

I. Suppose $N \equiv 0$. Then $f=(n, \lambda)(n, v-N)$. If $v \neq 0$, then $f=(n, \lambda v)$ $=\left(n, k^{2}-n\right)$. If $k^{2}-n \neq 0$, then $p$ divides neither argument, so $f=+1$; if $k^{2}-n \equiv 0$, then $n \equiv k^{2} \not \equiv 0$, so $f=\left(k^{2}, k^{2}-n\right)=+1$. If $v \equiv 0$, then $n \equiv k^{2}-\lambda v \equiv k^{2}$, so again $f=+1$.

II. Suppose $N \not \equiv 0$. Then $f=(n, \lambda)(n+\lambda N,-n N(v-N))$.

(1) If $n+\lambda N \equiv 0$, then $n \equiv-\lambda N$, so $\lambda \neq 0$. Hence $f=(n+\lambda N, \lambda(v-N))$. But $\lambda(v-N)=k^{2}-(n+\lambda N) \equiv k^{2}$, so if $v \neq N$ then $f=+1$. If $v \equiv N$, then $k \equiv 0$. Let $n+\lambda N=p^{a} n_{1}, k=p^{b} k_{1}$, where $n_{1}$ and $k_{1}$ are prime to $p$. Then $\lambda(v-N)=p^{2 b} k_{1}^{2}-p^{a} n_{1}$.

(a) If $a<2 b$, then $\lambda(v-N)=p^{a}\left(p^{2 b-a} k_{1}^{2}-n_{1}\right)$, so $f=(-1 \mid p)^{a}\left(n_{1} \mid p\right)^{a}\left(-n_{1} \mid p\right)^{a}$ $=+1$.

(b) If $2 b<a$, then $\lambda(v-N)=p^{2 b}\left(k_{1}^{2}-p^{a-2 b} n_{1}\right)$, so $f=(-1 \mid p)^{2 a b}\left(k_{1}^{2} \mid p\right)^{a}\left(n_{1} \mid p\right)^{2 b}$ $=+1$.

(c) If $a=2 b$, then $n+\lambda N=p^{2 b} n_{1}, \lambda(v-N)=p^{2 b}\left(k_{1}^{2}-n_{1}\right)$. If $k_{1}^{2}-n_{1} \not \equiv 0$, then it is easy to see that $f=+1$. If $k_{1}^{2}-n_{1} \equiv 0$, then $\lambda(v-N)=p^{c} d$, where $c>2 b$ and $d$ is prime to $p$. But then $f=(-1 \mid p)^{2 b c}\left(n_{1} \mid p\right)^{c}(d \mid p)^{2 b}=\left(n_{1} \mid p\right)^{c}=+1$, since $n_{1} \equiv k_{1}^{2} \not \equiv 0$.

(2) If $n+\lambda N \not \equiv 0$, then $f=(n, \lambda)(n+\lambda N, v-N)$. If $\lambda \equiv 0$, then $n+\lambda N \equiv n$, while $k^{2}-(n+\lambda N)=\lambda(v-N) \equiv 0$, so $k^{2} \equiv n+\lambda N \equiv n$, and hence $f=+1$. If $\lambda \not \equiv, v-N \equiv 0$, then $f=(n+\lambda N, v-N)$; but $n+\lambda N \equiv k^{2}$, so $f=+1$. 
For the case $n \equiv 0$, we will indicate an organization of the proof which will yield the desired result; it does not seem unlikely that there is a shorter and more elegant method of proof, however.

I. $k \equiv \lambda \equiv 0$.

(1) $N \equiv 0$,

(2) $N \not \equiv 0$

II. $k \equiv \lambda \not \equiv$.
(a) $N-1 \equiv 0$,
(b) $N-1 \not \equiv 0$.

(1) $v-N \equiv 0$,

(2) $N \equiv 0$,

(3) $N \not \equiv 0, v-N \not \equiv 0$.

Now since $c_{p}(C)=(m,-1)^{N(N+1) / 2}$, we have proved:

Theorem 3.1. If the $(v, k, \lambda)$ configuration $\pi$ possesses a standard collineation group (B) of order $m$, if $N$ points of $\pi$ are fixed by all of $(B)$, and if $n=k-\lambda$, $t=(v-N) / m$, then for each odd prime $p$ :

$$
(n,-1)_{p}^{(t+N-1) / 2}(n, m)_{p}^{N-1}(n, \lambda)_{p}=+1 .
$$

The condition of Theorem 3.1 can be rephrased in terms of Diophantine equations, where we note the following: the equation $x^{2}=a y^{2}+b z^{2}, a$ and $b$ nonzero integers, possesses a nontrivial solution in integers if and only if $(a, b)_{p}=+1$ for all primes $p$. Since the matrices $B$ and $C$ are positive definite, the case $p=2$ is trivial, and Theorem 3.1 allows us to assert:

THEOREM 3.2. If the $(v, k, \lambda)$ configuration $\pi$ possesses a standard collineation group (B) of order $m$, if $N$ points of $\pi$ are fixed by all of $(B)$, and if $n=k-\lambda$, $t=(v-N) / m, \epsilon=(t+N-1) / 2$, then the equation

$$
x^{2}=n y^{2}+(-1)^{\epsilon} m^{N-1} \lambda z^{2}
$$

possesses a nontrivial solution in integers.

In the statement of Theorem 3.2, nothing is said about the case when $t+N-1$ is not even; however, in this case $n$ must be a square, $n=n_{1}^{2}$, so $x=n_{1}, y=1, z=0$ is a nontrivial solution. Note further that we have only demonstrated Theorems 3.1 and 3.2 for $N \neq 0$, v. However, the results of $[4 ; 12]$ are easily seen to be equivalent in these cases (actually, the results of $[4 ; 12]$ are cast in a form similar to Theorem 3.2, rather than Theorem 3.1).

If the $(v, k, \lambda)$ configuration exists, then from [4], the condition $(n,-1)^{(v-1) / 2}(n, \lambda)=+1$ must also hold; using this, the above results can be somewhat simplified, as follows:

(i) If $N$ is even then either $n$ is a square or $m$ is odd and

$$
(n,-1)_{p}^{(m-1) / 2}(n, m)_{p}=+1 .
$$

(ii) If $N$ is odd and $m$ is even, then $(n,-1)_{p}^{\epsilon}=+1$, where $\epsilon=(v-N) / 2 m$. 
We omit the proofs of these statements; they are not difficult.

4. Finite projective planes. Now we specialize the configurations to the class of finite projective planes, which are the $(v, k, \lambda)$ configurations with $\lambda=1$. Suppose $(3$, of order $m$, is a standard collineation group of the projective plane $\pi$ of order $n$. The set of fixed points and lines of $\pi$ (with respect to (3) must be one of the following (see $[1 ; 5])$ :

Type (0). The empty set; then $N=0$.

Type (1). A line together with $n_{0}+1$ points on the line and $n_{0}$ further lines through one of these points; then $N=n_{0}+1$.

Type (2). A line $K$ together with $n_{0}$ points on $K$, one point $Q$ not on $K$, and the $n_{0}$ lines joining $Q$ to the $n_{0}$ fixed points on $K$; then $N=n_{0}+1$.

Type (3). A projective subplane $\pi_{0}$ of $\pi$, where $\pi_{0}$ has order $n_{0}$; then $N=n_{0}^{2}+n_{0}+1$.

Suppose $L$ is one of the lines fixed by all of $(B)$, and $L$ contains $x$ fixed points, and hence $n-x+1$ nonfixed points. Since no nonidentity element of (5) fixes any of these $n-x+1$ nonfixed points, we have $n-x+1 \equiv 0(\bmod m)$. In the respective cases, this is:

Type (1). $n \equiv n-n_{0} \equiv 0(\bmod m)$, or $n \equiv n_{0} \equiv 0(\bmod m)$.

Type (2). If $n_{0}=0$, then $n+1 \equiv 0(\bmod m)$. If $n_{0} \neq 0$, then $n-1 \equiv n-n_{0}+1$ $\equiv 0(\bmod m)$, or $n \equiv 1(\bmod m), n_{0} \equiv 2(\bmod m)$.

Type $(3) . n-n_{0} \equiv 0(\bmod m)$.

Although there are no fixed lines in type (0), it is clear that $m$ must divide $n^{2}+n+1$.

If $m=2$, then every point of $\pi$ is on a fixed line (see [1]), so according to which type occurs, we have: type (1), $n_{0}=n$; type (2), $n_{0}=n+1$; type (3), $n=n_{0}^{2}$. If $m$ is even, then the same conclusions must be valid, for otherwise (5) contains an element of order two which fixes points or lines of $\pi$ that are not fixed by all of $(5$.

Lemma 4.1. If $m$ is even then $N$ is odd. If $n$ is not a square, then if $n$ is even, $N=n+1$, while if $n$ is odd, then $N=n+2$.

Proof. If $m$ is even and type (3) occurs, then $N=n_{0}^{2}+n_{0}+1$ is odd. If $n$ is not a square, then type (3) does not occur; then according as $n$ is even or odd, type (1) or type (2), respectively, occur, whence $N=n+1$ or $N=n+2$.

Now suppose $m$ is even. If $n$ is a square, then Theorem 3.1 gives no information, so we assume that $n$ is not a square. If $n$ is even, then from Lemma 4.1, $(t+N-1) / 2=(n / 2)(n / m+1)$. If $n \equiv 0(\bmod 4)$, then $n / 2 \equiv 0(\bmod 2)$, while if $n \equiv 2(\bmod 4)$ then also $m \equiv 2(\bmod 4)$, so $n / m$ is odd and $n / m+1 \equiv 0$ $(\bmod 2)$. Hence $(t+N-1) / 2 \equiv 0(\bmod 2)$, and Theorem 3.1 gives no information.

If $n$ is odd, then $(t+N-1) / 2=[(n+1) / 2][(n-1) / m+1]$. If $n \equiv 3(\bmod 4)$ then $(n+1) / 2 \equiv 0(\bmod 2)$. If $n \equiv 1(\bmod 4)$, then $(t+N-1) / 2$ might be odd, but the existence conditions of [3] assert that $(n,-1)_{p}=+1$ if $\pi$ exists. So 
again Theorem 3.1 gives no information.

Thus for projective planes, all of the information of Theorem 3.1 is contained in the assertion (i) at the end of $\$ 3$ (and of course the existence conditions of [3] must also be satisfied).

As an example of the application of Theorem 3.1, we remark that it rejects everything it can for $n=10$; i.e., a projective plane of order 10 possesses only standard collineation groups which fix an odd number of points. On the other hand, Theorem 3.1 rejects nothing for $n=12$.

Theorem 3.1 can be applied to give information about planar ternary rings of certain special types (see $[5 ; 10 ; 14]$ for definition and discussion). Suppose $\pi$ is coordinatizable with a linear planar ternary ring with associative addition, where $\pi$ has order $n$ (in the terminology of Baer, this is a "Cartesian group"). Then the additive group and each of its subgroups make up standard collineation groups fixing the $n+1$ points on the "line at infinity," and fixing no other points. So if $n$ is odd, then $N=n+1$ is even.

THEOREM 4.1. If there is a linear planar ternary ring of odd order $n$ with associative addition, and if $m$ is any divisor of $n$ such that every group of order $n$ possesses a subgroup of order $m$ (e.g., $m$ is a prime divisor of $n$ ), then for all odd primes $p,(n,-1)_{p}^{(m-1) / 2}(n, m)_{p}=+1$.

Thus for instance, Theorem 4.1 rejects $n=15,35,45,51,65,75,85,91,99$, and gives no information for $n=39,55,63,95$.

Similarly, if $\pi$ possesses a linear planar ternary ring with associative multiplication, then the multiplicative group and each of its subgroups make up standard collineation groups fixing the $n+1$ points on the " $y$-axis" and one additional point. So if $n$ is even, then $N=n+2$ is even. Note that the multiplicative group has order $n-1$.

THEOREM 4.2. If there is a linear planar ternary ring of even order $n$ with associative multiplication, and if $m$ is any divisor of $n-1$ such that every group of order $n-1$ possesses a subgroup of order $m$, then for all odd primes $p$, $(n,-1)_{p}^{(m-1) / 2}(n, m)_{p}=+1$.

Theorem 4.2 rejects $n=10,26,34,40,50$, and gives no information for $n=12,18,20,24,28,36,44,48$.

5. Further investigations. The proof of Theorem 2.1 reveals properties of the sets $D_{i j}$ very similar to those possessed by difference sets and partial difference sets (see $[2: 6 ; 9 ; 10 ; 11]$ ). Indeed, the study of configurations (or planes) characterized by partial difference sets or difference sets is a specialization of the material here. It is not hard to construct a set of axioms for a group (\&) with subgroups $\mathfrak{P}_{i}, \Im_{j}$, subsets $D_{i j}, i, j=1,2, \cdots$, w, such that a $(v, k, \lambda)$ configuration can be constructed from the group; hence a collineation group, together with the appropriate subgroups and subsets, characterizes the configuration on which it acts. Since some powerful restrictions on 
groups with certain kinds of partial difference sets have been found, it is natural to inquire whether these restrictions will extend to the theory of more arbitrary collineation groups. In particular, theorems on "multipliers" would be of great interest: essentially, this is asking that the existence of certain collineations be shown to imply the existence of further collineations. For instance, the proof of Theorem 3.1 of [2] can be generalized easily to prove the following:

THEOREM 5.1. If (S) is a collineation group of the $(v, k, \lambda)$ configuration $\pi, N(\$)$ the normalizer of $\&$ in the group of all collineations of $\pi$, and if the $D_{i j}$ are defined as in $\$ 2$, then a necessary and sufficient condition that the mapping $T$ of $\pi$ be a collineation of $\pi$ contained in $N(\mathbb{S})$ is that

$$
\left(P_{i} x\right) T=P_{i \alpha} a_{i}^{-1} \cdot x \theta, \quad\left(J_{i} x\right) T=J_{i \beta} b_{i} \cdot x \theta,
$$

for all $x \in(S)$ and for all $i=1,2, \cdots, w$, where $\alpha$ and $\beta$ are permutations of the set $(1,2, \cdots, w)$ and $\theta$ is an automorphism of (B) such that $D_{i j} \theta=a_{i} D_{i \alpha, j \beta} b_{j}$, for all $i, j$.

A mapping $\theta$ with the properties given in Theorem 5.1 might be called a multiplier, but it is not clear what (if anything) should correspond to the more fruitful notion of right multiplier, as in [2]. In particular, what is the meaning of the group $N(\$) /(\$)$ ?

Theorem 2.2 is quite properly a generalization of the incidence malrix equations of earlier papers $[3 ; 4]$, and for $m=1$ includes those earlier relations. An added difficulty exists here, however: even if an integral matrix $A$ can be determined (and the conclusion of Theorem 3.1, for instance, only assures us that a rational $A$ can be found), the sets $D_{i j}$ must still be shown to exist if the $(v, k, \lambda)$ configuration is to be constructed.

Added in proof. (1) In a paper (Generalized incidence matrices over group algebras) to appear shortly in the Illinois Journal of Mathematics, the author has shown that the matrix equations of Theorem 2.2 can be deduced from more general equations involving group algebra matrices, and has proved from these latter equations that if $\lambda=1, n \equiv 2(\bmod 4)$, then $\pi$ possesses no collineations of even order. The group algebra matrix equations have the further property that their existence is equivalent to the existence of the design with the specified collineation group.

(2) H. P. Dembowski, in a doctoral dissertation at Frankfort au Main, has derived more general results very similar to those in this paper, concerning so-called "tactical decompositions."

\section{BIBLIOGRAPHY}

1. Reinhold Baer, Projectivities with fixed points on every line of the plane, Bull. Amer. Math. Soc. vol. 52 (1946) pp. 273-286.

2. R. H. Bruck, Difference sets in a finite group, Trans. Amer. Math. Soc. vol. 78 (1955) pp. 464-481. 
3. R. H. Bruck and H. J. Ryser, The non-existence of certain finite projective planes, Canadian Journal of Mathematics vol. 1 (1949) pp. 88-93.

4. S. Chowla and H. J. Ryser, Combinatorial problems, Canadian Journal of Mathematics vol. 2 (1950) pp. 93-99.

5. Marshall Hall, Jr., . .jective planes, Trans. Amer. Math. Soc. vol. 54 (1943) pp. 229277.

6. —_- Cyclic projective planes, Duke Math. J. vol. 14 (1947) pp. 1079-1090.

7. - - Projective planes and related topics, California Institute of Technology, 1954.

8. Marshall Hall, Jr. and H. J. Ryser, Cyclic incidence matrices, Canadian Journal of Mathematics vol. 3 (1951) pp. 495-502. 295-301.

9. A. J. Hoffman, Cyclic affine planes, Canadian Journal of Mathematics vol. 4 (1952) pp.

10. D. R. Hughes, Planar division neo-rings, Trans. Amer. Math. Soc. vol. 80 (1955) pp. 502-527.

11. - Partial difference sets, Amer. J. Math. vol. 78 (1956) pp. 650-674.

12. - - Regular collineation groups, Proc. Amer. Math. Soc. vol. 8 (1957) pp. 165-168.

13. B. W. Jones, The arithmetic theory of quadratic forms, Carus Math. Monographs, no. 10.

14. Günter Pickert, Projektive Ebenen, Berlin, 1955.

15. H. J. Ryser, A note on a combinatorial problem, Proc. Amer. Math. Soc. vol. 1 (1950) pp. $422-424$.

16. - Matrices with integer elements in combinatorial investigations, Amer. J. Math. vol. 74 (1952) pp. 769-773.

The Ohio State University, Columbus, Ohio 